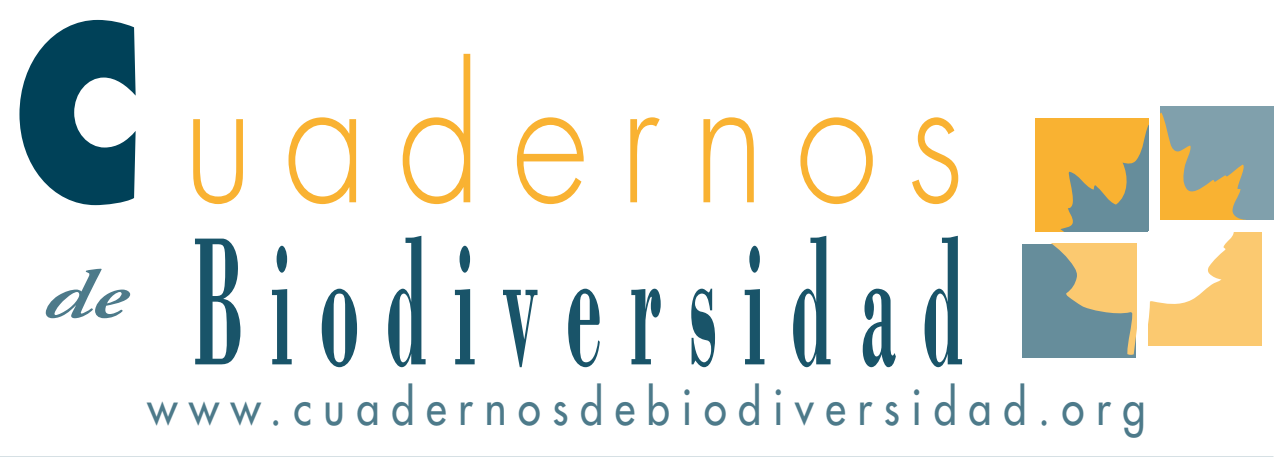

\title{
Una mirada a la dispersión de semillas en las excretas de mamíferos
}

\author{
R. Medrano Nájera', M. Ramírez Pinero ${ }^{2}$, S. Guevara Sada ${ }^{2}$ \\ i Instituto Tecnológico de Conkal, Conkal, Yucatán. MéXico \\ 2 Instituto de Ecología A. C. Xalapa, Veracruz, México \\ MAYITZA.RAMIREZ@GMAIL.COM
}

\section{ABSTRACT}

A look at seed dispersal via mammal droppings. Terrestrial mammals are good dispersers of the fruits and seeds of seasonal rainforest in the Gulf of Mexico. Their importance lies in the huge quantity of fruit and seeds they consume, and their daily and seasonal movement through natural and secondary vegetation and agricultural fields. Their importance is even more noteworthy in the face of increasing deforestation and forest fragmentation, which limits the dispersal of propagules across the landscape and affects, among other things, the potential for forest regeneration.

To evaluate fruit and seed consumption and dispersal over 12 months, the droppings of rainforest mammals were analyzed for the rainforest and an abandoned pasture $(8,400 \mathrm{~m} 2$ respectively) on the coast of central Veracruz, Mexico. In the rainforest 158 droppings were collected and 47 in the pasture from a total of eight mammal species, six of which are known dispersers. The droppings contained 150 seeds belonging to 36 species and 19 families. The deposition of different species and quantities of seeds in the forest and pasture indicates that mammals disperse more rainforest species to the pasture than vice versa.

Key words: seed dispersal, species availability, natural regeneration, terrestrial mammals, coastal landscape 


\section{RESUMEN}

Los mamíferos terrestres son buenos dispersores de frutos y semillas de la selva estacional en la costa del Golfo de México. Deben su importancia a que consumen gran cantidad de frutos y semillas, al largo tiempo que los mantienen en su tracto digestivo y a su movilidad diaria y estacional a través de la vegetación natural, secundaria y campos agropecuarios. Su importancia resalta ante la creciente deforestación y fragmentación de la selva, que limita la dispersión de los propágulos en el paisaje, y que afecta entre otras cosas, al potencial de regeneración de la selva.

Con el objetivo de valorar el consumo de frutos y semillas y su dispersión, se analizaron durante 12 meses las excretas de mamíferos en la selva y en un potrero abandonado $(8.400 \mathrm{~m} 2$ respectivamente), en la costa del centro de Veracruz. La colecta de 158 excretas en la selva y 47 en el pastizal, correspondieron a 8 especies de mamíferos, aunque solo 6 fueron los dispersores. Las excretas contuvieron 150 semillas de 36 especies de 19 familias. La deposición de distintas especies y cantidades de semillas en la selva y el pastizal indican que los mamíferos dispersan más especies de la selva al pastizal que viceversa.

Palabras clave: dispersión de semillas, disponibilidad de especies, regeneración natural, mamíferos terrestres, paisaje costero.

\section{INTRODUCCIÓN}

El paisaje costero del Estado de Veracruz, México, se puede describir como un mosaico formado de fragmentos de selva baja caducifolia, selva mediana subcaducifolia, selva baja perennifolia inundable, manglar, palmar, vegetación riparia, vegetación de dunas costeras, humedal y pastizal (MorenoCasasola, 2006) (Figs. 1 y 2).

En este mosaico destaca la selva baja caducifolia, (sensu Miranda y Hernández, 1961), como el ecosistema predominante. Sus especies están presentes en otros componentes del paisaje, y participan activamente en la sucesión secundaria y en la regeneración de la vegetación (Guevara, 2006; Guevara y Moreno-Casasola, 2008).

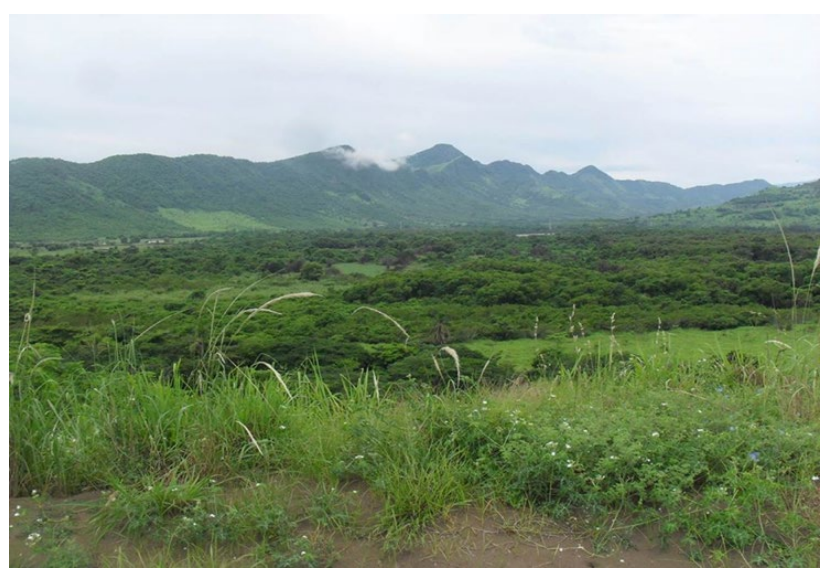

Figura 1. El paisaje costero del centro de Veracruz se extiende en la planicie costera hasta las laderas de la sierra de Manuel Díaz

La diseminación de las especies de la selva en el paisaje se debe a la variedad de síndromes de dispersión que poseen: anemócora, hidrócora, zoócora, sin embargo la deforestación y la fragmentación de la selva la sesgan y la restringen.

Es sabido que en las selvas tropicales húmedas y secas, los vertebrados terrestres y voladores consumen y dispersan numerosas especies en cantidades considerables, tanto, que se les podría considerar como diseñadores del paisaje (Herrera, 1985). Su importancia varía con el tipo de selva de que trate, pero en promedio se estima que entre 51 y $98 \%$ del total de las especies arbóreas y leñosas son diseminadas por los vertebrados (Galindo, 1998; Entwistle y Dunstone, 2000).

En las regiones tropicales las aves y los murciélagos, son los principales responsables de la dispersión de semillas (Guitián et al., 2010 y Stoner et al., 2007). Ambos son gremios que se adaptan fácilmente a los cambios en el paisaje ocasionados por la deforestación y la fragmentación (López-Bao y González-Varo, 2011; Zhou et al., 2008; Koike et al., 2008; Wang et al., 2007; Asquit et al., 1997; Herrera, 1985).

Los murciélagos, en particular, se alimentan de numerosas especies de plantas, sus poblaciones son grandes y tienen una gran capacidad de vuelo, especialmente a través de los campos abiertos. A su lado, aunque han sido muy poco tomados en cuenta, están algunas especies de mamíferos terres- 
tres omnívoros y algunos carnívoros facultativos (Koike et al., 2008), cuyos hábitos los hacen buenos dispersores; consumen frutos y semillas inasequibles a las aves y murciélagos, retienen largo tiempo las semillas en su tracto digestivo escarificándolas, se desplazan cotidiana y estacionalmente en el paisaje y la deposición de sus excretas responde a condiciones peculiares (López-Bao y González-Varo, 2011, Matías et al., 2009).

La mayoría de las especies de mamíferos terrestres habitan en la selva, sin embargo en su búsqueda de alimento recorren continuamente sitios deforestados y campos de cultivo (Matías et al., 2009), una rutina probablemente asociada a la forma de uso agrícola del suelo en Mesoamérica, que se mantiene a pesar de los drásticos cambios actuales del paisaje. En la década de 1990 la selva baja caducifolia del centro de Veracruz, tuvo una tasa anual de deforestación de $4.33 \%$; estimándose que actualmente solo queda el $7 \%$ de la vegetación original, de la cual un tercio es vegetación secundaria (Ramírez-Pinero, 2012).

Ante este panorama, se puede aseverar que el mantenimiento de la biodiversidad del paisaje de la selva estacional depende de la eficiente dispersión de las plantas y los animales entre los fragmentos y a través de los campos agrícolas y ganaderos.

El objetivo de este estudio fue determinar las especies que son dispersadas por los mamíferos terrestres a través de sus excretas, en la selva baja caducifolia considerada fuente de propágulos y un pastizal abandonado considerado receptor de los propágulos.

\section{METODOLOGÍA}

El estudio se llevó a cabo en el área natural protegida "Cansaburro" perteneciente a la asociación civil PRONATURA, ubicada en la planicie costera del Golfo de México, Municipio de Actopan, centro del estado de Veracruz (Figura 2). El predio está en dunas de arena paralelas a la costa, con altura máxima de $80 \mathrm{msnm}$ y una pendiente entre $4^{\circ}$ y $60^{\circ}$.

En la ladera de sotavento de las dunas se encuentra un fragmento de selva con árboles de la selva mediana subcaducifolia como Enterolobium cyclocarpum, Ficus cotinifolia y Pouteria hypoglauca y con especies de selva baja caducifolia como Diphysa americana, Acacia cornigera, Jatropha curcas, Psychotria erythrocarpa y Bursera simaruba, que se prolonga hacia los humedales de la laguna de La Mancha (Figs. 1 y 2).

La ladera de barlovento está cubierta por un pastizal de especies nativas salpicado con arbustos de Opuntia stricta y árboles de Diphysa americana (Castillo-Campos, 2005), que fue utilizado para vacas y abandonado hace aproximadamente tres años (Fig. 3).

En cada ladera se delimitó una parcela de 8.400 $\mathrm{m}^{2}(60 \times 140 \mathrm{~m})$, que se recorrieron mensualmente durante un año (febrero 2012 a enero de 2013), para registrar las huellas y rastros de mamíferos y colectar sus excretas. Se recogieron los pelos de mamífero encontrados alrededor de las excretas para identificar las especies (Colección Taxonómica del Instituto de Investigaciones Biológicas de la Universidad Veracruzana; Baca y Sánchez-Cordero, 2004; Juárez et al., 2009; Pech-Canche et al., 2009, Martínez-Vázquez et al., 2010).

Se extrajeron las semillas contenidas en cada excreta se dejaron secar, se identificaron y se contaron (Grajales, 2003, Martínez-Vázquez et al., 2010), y algunas se pusieron a germinar en un invernadero del Centro de Investigaciones Costeras de La Mancha CICOLMA del Instituto de Ecología, A.C.

El clima del año de muestreo, fue notablemente más húmedo que la media de los últimos 30 años de este sitio. La temporada húmeda se extendió de junio a octubre y la temporada seca de noviembre a mayo (Fig. 4). 


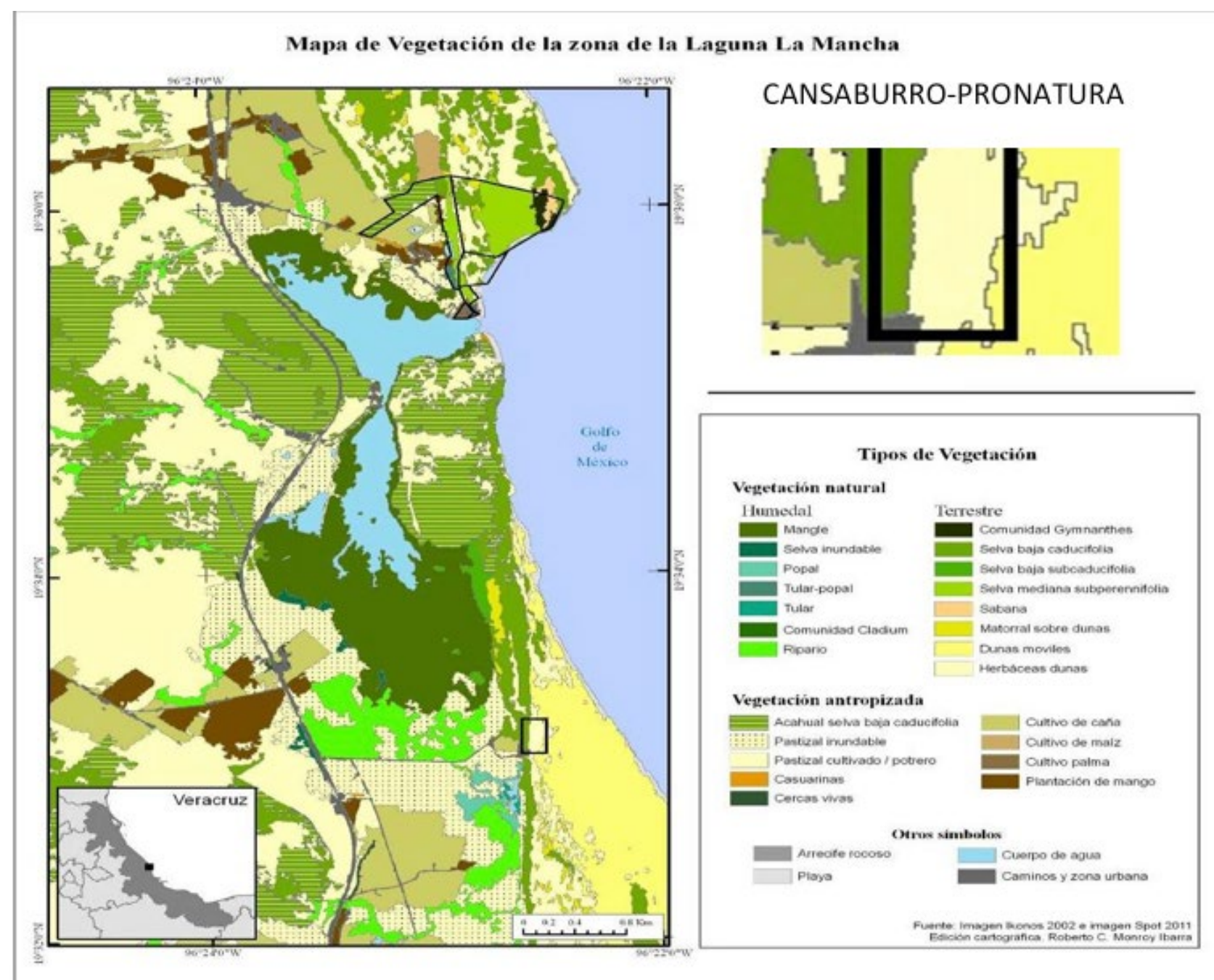

Figura 2. Mapa de la vegetación y uso del suelo de la zona de estudio. El predio "Cansaburro" está indicado por el rectángulo negro

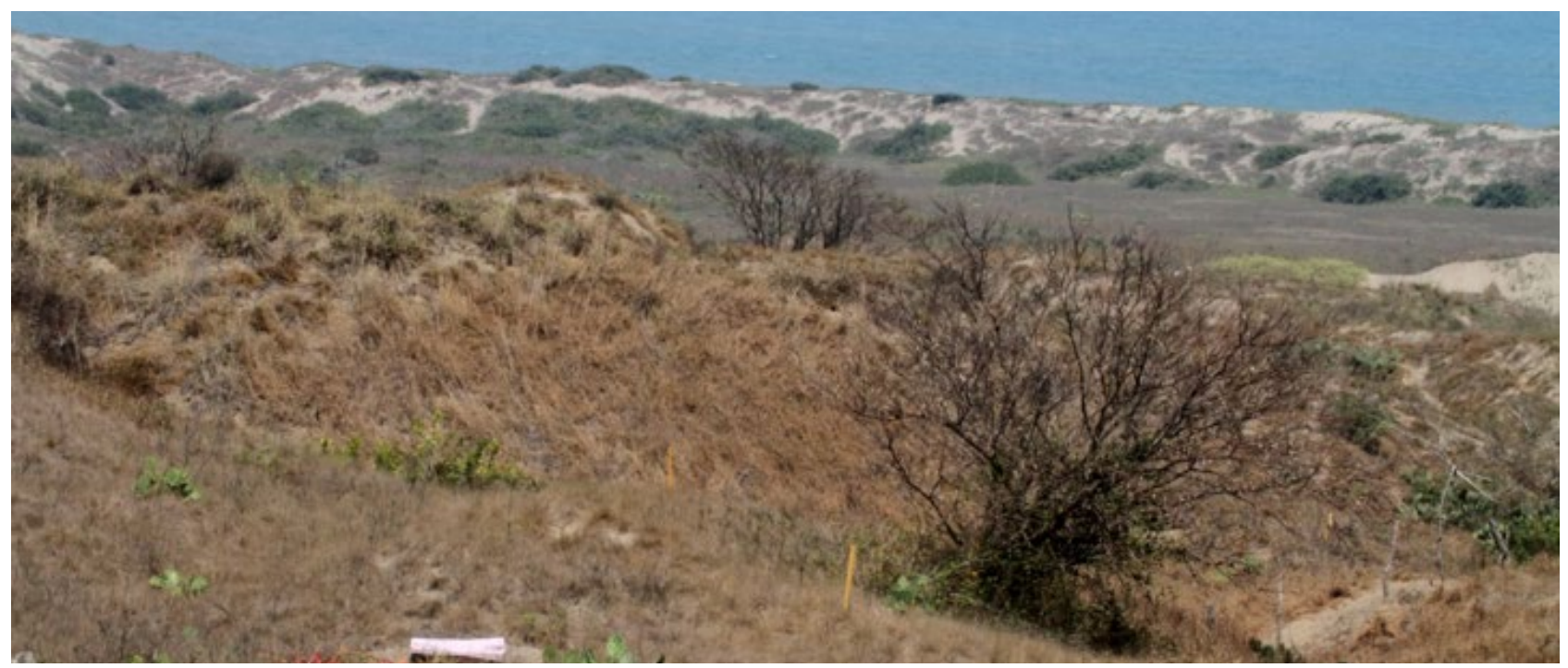

Figura 3. Pastizal abandonado en la ladera de barlovento de las dunas costeras 


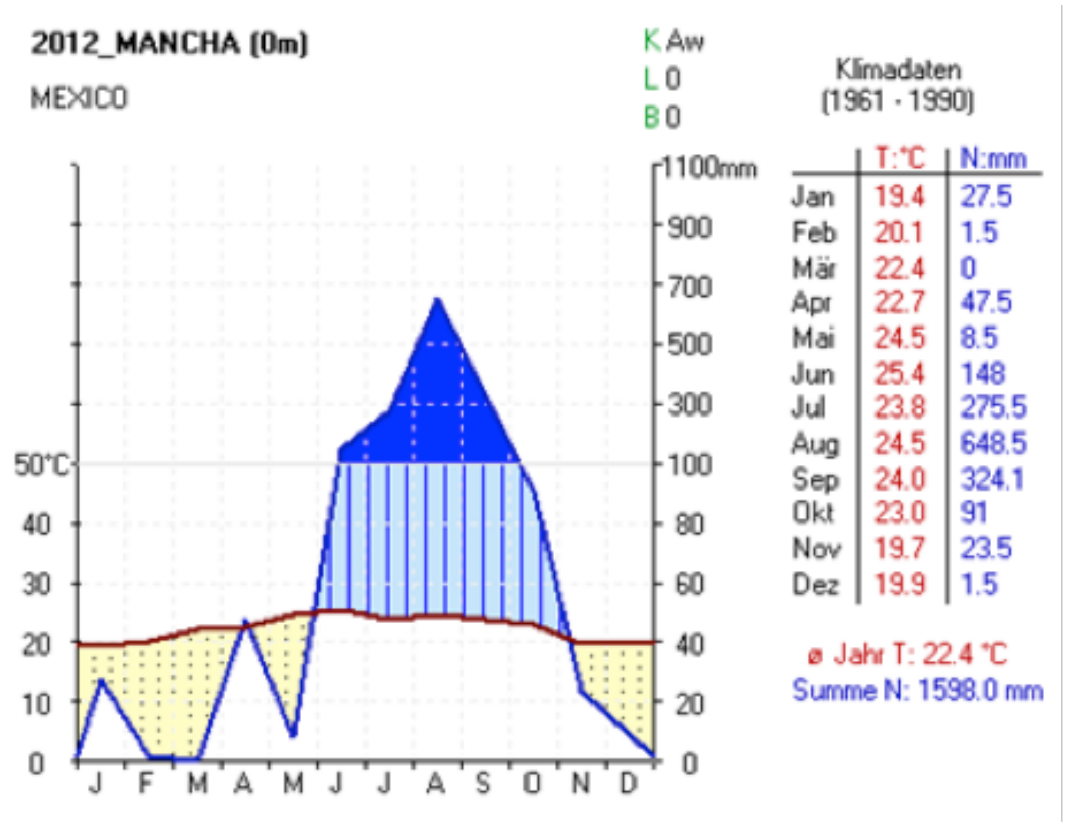

Figura 4. Climograma, año 2012. La línea roja indica la temperatura mensual promedio y la línea azul la precipitación. En azul claro se indica los meses de lluvias y en azul obscuro la temporada húmeda (disponibilidad de agua en el suelo). En amarillo se indica la temporada seca

\section{RESULTADOS}

Se colectó un total de 205 excretas y se identificaron ocho especies de mamíferos con hábitos carnívoros y omnívoros. El mayor número de excretas se encontró durante la temporada seca: 115 excretas en la selva y 43 excretas en el pastizal. Durante la temporada húmeda el número de excretas disminuyó a 43 excretas en la selva y 4 excretas en el pastizal (Tabla 1). El número promedio mensual de excretas fue de 14.3 en la selva y 4.2 en el pastizal.

El número total de semillas en las excretas fue de 48.791, de 36 especies de 19 familias. La mayor cantidad se encontró en las excretas de la selva durante la temporada seca $(30.919 / 8.400 \mathrm{~m} 2)$ y el número menor en el pastizal $(385 / 8.400 \mathrm{~m} 2)$ durante la temporada húmeda (Tabla 1 y Fig. 5).

\begin{tabular}{|c|c|c|c|c|}
\hline & \multicolumn{2}{|c|}{$\begin{array}{l}\text { Temporada seca } \\
\text { Precipitación } \\
\text { acumulada de } \\
\text { noviembre a mayo } \\
110 \mathrm{~mm}\end{array}$} & \multicolumn{2}{|c|}{$\begin{array}{c}\text { Temporada } \\
\text { húmeda } \\
\text { Precipitación } \\
\text { acumulada } \\
\text { de junio a octubre } \\
\text { 1,487 mm }\end{array}$} \\
\hline excretas & $\begin{array}{c}\text { selva } \\
(56 \%) \\
115\end{array}$ & $\begin{array}{c}\text { pastizal } \\
(21 \%) \\
43\end{array}$ & $\begin{array}{c}\text { selva } \\
(21 \%) \\
43\end{array}$ & $\begin{array}{c}\text { pastizal } \\
(2 \%) \\
4 \\
\end{array}$ \\
\hline semillas & $\begin{array}{c}(63 \%) \\
30,919\end{array}$ & $\begin{array}{l}(27 \%) \\
13,321\end{array}$ & $\begin{array}{l}(9 \%) \\
4,166\end{array}$ & $\begin{array}{c}(1 \%) \\
385\end{array}$ \\
\hline especies & $\begin{array}{c}(83 \%) \\
30\end{array}$ & $\begin{array}{c}(42 \%) \\
15\end{array}$ & $\begin{array}{c}(39 \%) \\
14\end{array}$ & $\begin{array}{c}(19 \%) \\
7\end{array}$ \\
\hline
\end{tabular}




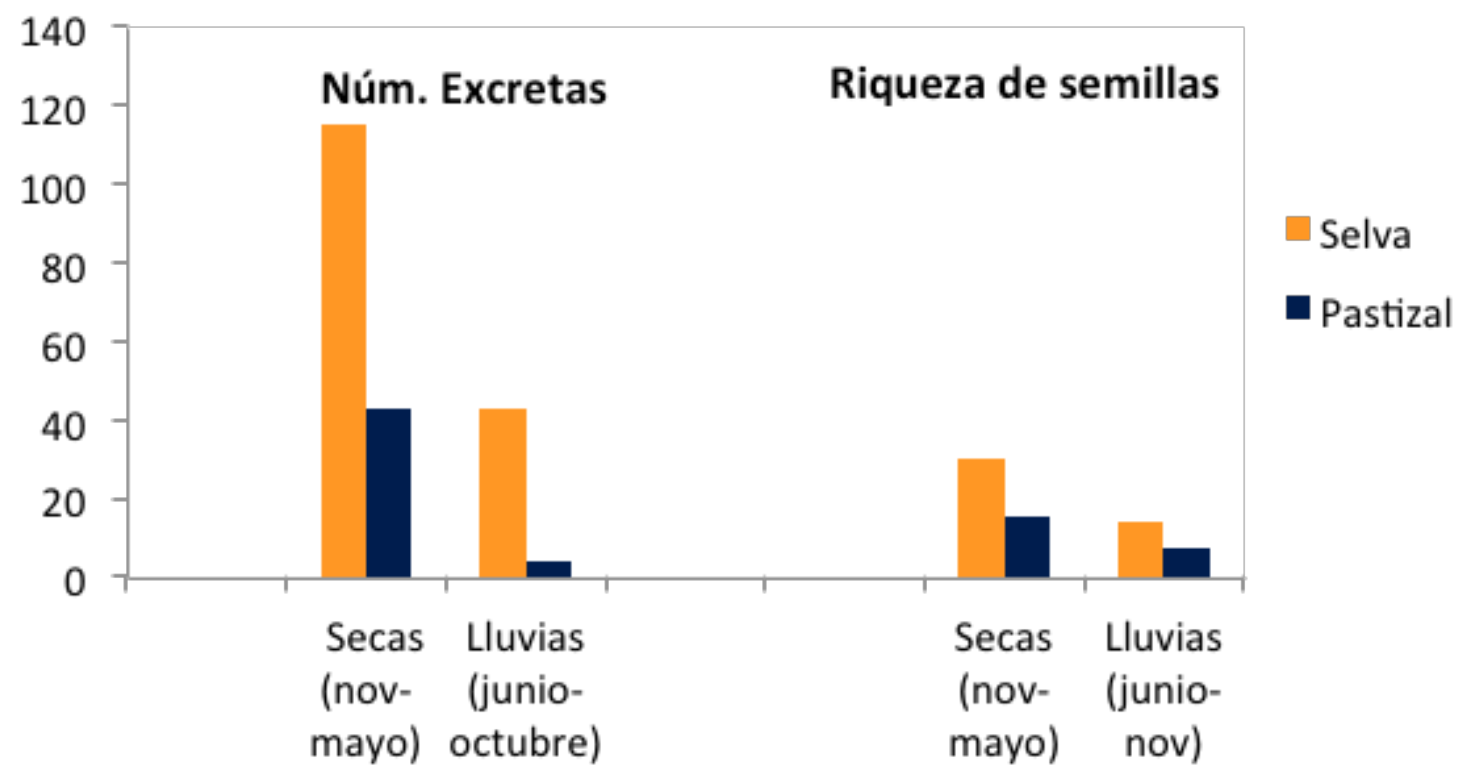

Figura 5. Número de excretas y riqueza por temporada en cada parcela $(8400 \mathrm{~m} 2)$

En la selva se obtuvo un total de 32 especies de semillas y en el pastizal 20 especies. La selva y el pastizal compartieron 16 especies; otras 16 especies se encontraron exclusivamente en la selva y 4 especies se encontraron exclusivamente en el pastizal.

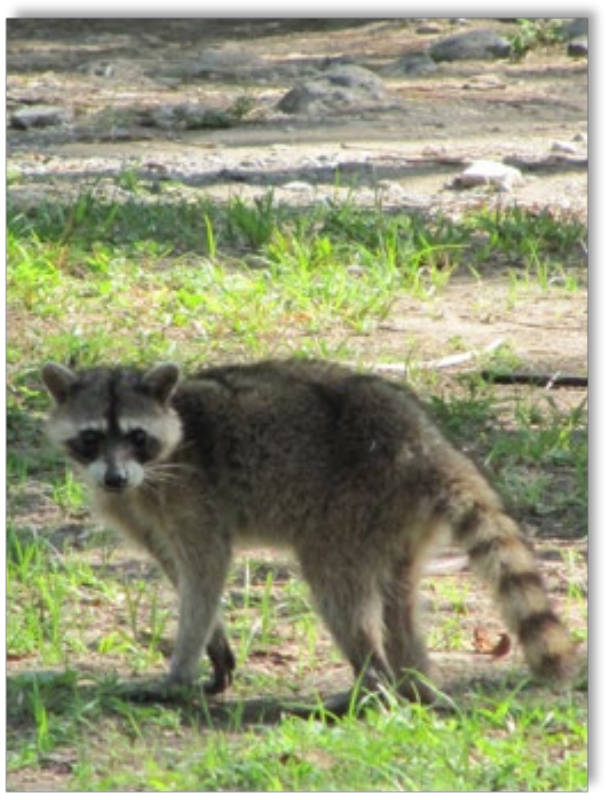

Mapache, Procyon lotor (Linnaeus, 1758)
En el pastizal se identificaron dos especies de mamíferos dispersores: el Coyote (Canis latrans Say, 1823) y la Zorra gris (Urocyon cinereoargenteus Schreber, 1775). En la selva se identificaron seis especies de mamíferos: Zarigüeya (Didelphis sp.), Onza (Puma yagouaroundi Étienne Geoffroy SaintHilaire, 1803), Cacomixtle (Bassariscus sp), Mapache (Procyon lotor (Linnaeus, 1758)), Armadillo común (Dasypus novemcinctus Linnaeus, 1758) y Oso hormiguero (Tamandua mexicana Saussure, 1860). Tabla 2.

Entre estas 8 especies, se identificaron cuatro (Zorro, Cacomixtle, Mapache y Armadillo común) como dispersores de semillas entre la selva y el pastizal abandonado. El Coyote y la Zorra gris, dispersan frutos y semillas pero solo en el pastizal y acaso en los bordes de la selva, pues habitan en campos abiertos y no penetran a la selva; la Onza es carnívora aunque eventualmente salga de la selva y el Oso hormiguero es un consumidor estricto de hormigas (Tabla 2).

Las semillas contenidas en las excretas de las cuatro especies de omnívoros muestran que consumen frutos y semillas de 36 especies de plantas, de las cuales dispersan cuando menos 16 desde la 
Tabla 2. Especies de mamíferos identificados en el pastizal y la selva de Cansaburro. El tipo de alimentación y la situación de las especies: $\mathrm{P}=$ peligro de extinción, $\mathrm{A}=$ amenazada, fueron tomadas de la Norma Oficial Mexicana. NOM-059-SEMARNAT-2001

\begin{tabular}{|c|c|c|c|c|}
\hline Nombre común & Familia & Especie & Tipo de alimentación & $\begin{array}{l}\text { Situación de la } \\
\text { especie }\end{array}$ \\
\hline & & PASTIZAL & & \\
\hline $\begin{array}{l}\text { Coyote, Perro de } \\
\text { monte }\end{array}$ & Canidae & Canis latrans & Omnívora & - \\
\hline \multirow[t]{2}{*}{$\begin{array}{l}\text { Zorra, Zorra gris, } \\
\text { Gato de monte }\end{array}$} & Canidae & $\begin{array}{l}\text { Urocyon } \\
\text { cineareoargenteus }\end{array}$ & Omnívora & - \\
\hline & & SELVA & & \\
\hline $\begin{array}{l}\text { Jaguarundi, Onza, } \\
\text { León miquero }\end{array}$ & Felidae & Puma yagouaroundi & Carnívora oportunista & A \\
\hline $\begin{array}{l}\text { Zorro, Comadreja, } \\
\text { Zarigüeya }\end{array}$ & Didelphidae & Didelphis sp. & Omnívora & - \\
\hline Cacomixtle & Procyonidae & Bassariscus sp. & Omnívora & A \\
\hline $\begin{array}{l}\text { Mapache, Osito } \\
\text { lavador }\end{array}$ & Procyonidae & Procyon lotor & Omnívora & - \\
\hline $\begin{array}{l}\text { Armadillo común, } \\
\text { Mulita, Toche }\end{array}$ & Dasypodidae & Dasypus novemcinctus & Insectívoro omnívoro & - \\
\hline $\begin{array}{l}\text { Oso hormiguero, } \\
\text { Brazo fuerte }\end{array}$ & Myrmecophagidae & Tamandua mexicana & $\begin{array}{l}\text { Insectívora (hormigas } \\
\text { y termitas) }\end{array}$ & $\mathrm{P}$ \\
\hline
\end{tabular}

selva al pastizal. La selva es la fuente principal de propágulos como revelan las 32 especies encontradas en las excretas y el pastizal es el receptor de las semillas como señalan las 16 especies que comparte con la selva.

La mayoría de las especies dispersadas por los mamíferos son árboles y arbustos, de la selva. Entre las 16 especies de plantas compartidas entre la selva y el pastizal destacan algunas que son características del estrato arbustivo y arbóreo de la selva baja caducifolia como: Rhamnus humboldtiana, Chiococca alba, Crossopetalum uragoga, Eugenia capuli, Psychotria erythrocarpa, Schaefferia frutescens, Parmentiera aculeatea y otras provenientes del dosel de la selva mediana subcaducifolia como: Ficus cotinifolia, Ficus obtusifolia, Manilkara zapota, Ehretia tinifolia y Pouteria glomerata, lo que significa una sensible contribución a la disponibilidad de semillas del pastizal en vista de su regeneración.

Algunas especies como Chiococca alba y Parmentiera aculeatea, un arbusto y árbol pequeño respectivamente, son de rápido crecimiento y demandantes de luz, se establecen en el pastizal actuando como "nodrizas" para especies de la selva que son dependientes de la sombra y de lento crecimiento.

Con el fin de tener una idea acerca de la viabilidad de las semillas extraídas de las excretas, las especies más frecuentes o destacadas por su tamaño o número, se pusieron a germinar sobre arena húmeda en el invernadero. La cantidad de semillas germinadas varió, como ocurrió con Manilkara zapota, de la 
que sólo se obtuvieron 2 semillas por ser un árbol de la selva y tener una semilla de gran tamaño (Tabla 3).

Germinaron semillas de todas las especies probadas, demostrando que mantienen su viabilidad a pesar del paso por el tracto digestivo y su permanencia en el suelo. El porcentaje de germinación fue bajo, tal vez debido a que el tamaño de muestra fue pequeńo y a que las condiciones de germinación en el invernadero no fueron óptimas.

Tabla 3. Cantidad de semillas y porcentaje de germinación

\begin{tabular}{l|c|c}
\multicolumn{1}{c}{ Especie } & $\begin{array}{c}\text { Número } \\
\text { de semillas } \\
\text { probadas }\end{array}$ & $\begin{array}{c}\text { Porcentaje de } \\
\text { germinación }\end{array}$ \\
\hline Randia laetevirens & 120 & 38 \\
\hline Eugenia capuli & 131 & 29 \\
\hline Parmentiera aculeata & 36 & 28 \\
\hline Ficus cotinifolia & 216 & 21 \\
\hline Solanum sp. & 65 & 15 \\
\hline Guazuma ulmifolia & 74 & 12 \\
\hline Manilkara zapota & 2 & 100
\end{tabular}

\section{DISCUSIÓN}

La movilidad de los mamíferos terrestres en el paisaje costero tiene resultados positivos para la regeneración de sitios perturbados, pues llevan semillas de un sitio a otro. Contribuyeron con 16 especies de semillas al acervo de especies que eventualmente participan en el proceso de sucesión secundaria en el pastizal abandonado. Aunque hay que considerar que las semillas de las excretas pueden ser posteriormente dispersadas por cangrejos, hormigas o coleópteros, hasta donde haya condiciones de germinación y establecimiento o que induzcan latencia incorporándolas al banco de semillas del suelo.
Los mamíferos terrestres efectivamente se desplazan en el paisaje cotidianamente como muestra la variabilidad de los muestreos mensuales, y estacionalmente como lo demuestra la acumulación de excretas y la mayor abundancia y riqueza de semillas durante la temporada seca (Guitián et al., 2010), la cual coincide con la mayor disponibilidad de frutos de noviembre a abril (Ramírez-Pinero, 2012) Tabla 1.

El patrón de desplazamiento de cada especie de mamífero depende de su fisionomía (Perea et al., 2013), del comportamiento ante sus depredadores (Gehring et al., 2002), de las características de la matriz del paisaje, de los elementos del paisaje que facilitan su desplazamiento entre hábitats y de la oferta de recursos alimenticios vegetales y animales en cada sitio.

Los mamíferos frecuentan sitios perturbados o desprovistos de vegetación (Peredo et al., 2013); el pastizal puede atraerles por la cantidad de cangrejos, pequeños reptiles o insectos que complementan su dieta.

Una de las grandes diferencias de la dispersión de semillas por aves y murciélagos y de los mamíferos terrestres es la cantidad de semillas y el sitio donde se depositan las excretas en el paisaje "seed shadow" (Koike et al., 2008 y Zouh et al., 2008). Las excretas de aves y murciélagos son numerosas y se concentran alrededor de sitios de percheo, o bajo árboles.

En este estudio la mayoría de las excretas que se encontraron en el pastizal estaban en sitios desprovistos de vegetación, principalmente en caminos o veredas. En la selva se observó que dejaban las excretas en sitios fijos "letrinas", bajo la copa de algunos árboles como Ficus cotinifola, donde se encontraron semillas de Celtis iguanaea, Eugenia sp. y Annona reticulata. Sin embargo, el patrón, como se explicó antes, se puede modificar por el efecto de dispersores secundarios.

Por todas éstas características y en vista de que la mayoría de las especies que dispersan desde la selva hacia el pastizal son árboles y arbustos, podemos 
considerar a los mamíferos terrestres como potenciales "restauradores" (Matías et al., 2008) de paisajes fragmentados y transformados.

Sin embargo, todavía es necesario entender cómo se adaptan los mamíferos terrestres a los cambios que implica la fragmentación del paisaje, cómo interactúan con las especies vegetales y qué determina sus patrones de deposición de semillas.

La existencia de especies frugívoras y la dispersión activa de semillas son una garantía del mantenimiento de la biodiversidad del paisaje costero a largo plazo.

\section{REFERENCIAS}

Asquith, N. M., Wright, S. J. \& Clauss, M. J. 1997. Does mammal community composition control recruitment in neotropical forests? Evidence from Panama. Ecology 78:941-946

Baca, I. \& Sánchez-Cordero, V. 2004. Catálogo de pelos de guardia dorsal en mamíferos del estado de Oaxaca, México. Anales del Instituto de Biología. Serie Zoología 75: 383-437.

Castillo-Campos, G. 2005. Árboles y arbustos de la Reserva Natural de la Mancha, Veracruz. Instituto de Ecología A.C. México. 143 pp.

Entwistle, A. \& Dunstone. N. 2000. Priorities for the conservation of mammalian diversity: has the panda had its day? Cambridge University Press. United Kingdom. 270 pp.

Galindo, G. J. 1998. Dispersión de semillas por murciélagos: su importancia en la conservación y regeneración del bosque tropical. Acta Zoológica Mexicana (nueva serie), número 73; Instituto de Ecología. A.C.

Gehring, T. M., \& Swihart, R. K. 2003. Body size, niche breadth, and ecologically scaled responses to habitat fragmentation: mammalian predators in an agricultural landscape. Biological Conservation.109:283-295.

Grajales, K. M., Rodríguez-Estrella, R. \& Hernández, J. C. 2003. Dieta estacional del coyote Canis latrans durante el periodo 1996-1997 en el Desierto de Vizcaíno, Baja California Sur, México. Acta Zoologica Mexicana (nueva serie), 89, 17-28.
Guevara, S. 2006. El paisaje del viento. En Moreno-Casasola, P. (ed) Entornos veracruzanos: la costa de La Mancha. Instituto de Ecología, A.C., México, 576 pp.

Guevara, S. \& Moreno-Casasola P. 2008. 30 años en el paisaje costero veracruzano. Instituto de Ecología, A.C. y Comisión Federal de Electricidad, México, 240 pp.

Guitián, J. \& Munilla, I. 2010. Responses of mammal dispersers to fruit availability: Rowan: (Sorbus aucuparia) and carnivores in mountain habitats of northern Spain. Acta Oecologica 36:242-247.

Herrera, C. M. 1985. Determinants of plant-animal coevolution: the case of mutualistic dispersal of seeds by vertebrates. Oikos 44:132-141.

Juárez, D. Bustamante, M. Estrada, C. López, J. Moreira, J. \& Quintana, Y. 2009. Guía ilustrada de pelos para la identificación de mamíferos mayores y medianos de Guatemala. Universidad de San Carlos Guatemala. Guatemala.

Koike, S., Morimoto, H., Goto, Y., Kozakai, C. \& Yamazaki, K. 2008. Frugivory of carnivores and seed dispersal of fleshy fruits in cool-temperate deciduous forests. Journal of Forest Research 13:215-222.

López-Bao, J. V., \& González-Varo, J. P. 2011. Frugivory and spatial patterns of seed deposition by carnivorous mammals in anthropogenic landscapes: a multi-scale approach. PloS one 6:e14569.

Martínez-Vázquez J., Gonzáles-Monroy R. M. \& Díaz-Díaz, D. 2010. Habitos alimenticios del coyote en el parque nacional pico de Orizaba. Estudio mastofaunístico del Parque Nacional La Malinche, Taxcala, México. Therya. Vol. 1(2): 145-154.

Matías, L., Zamora, R. Mendoza, I. \& Hódar, J. A. 2008. Seed dispersal patterns by large frugivorous mammals in a degraded mosaic landscape. Restoration Ecology 18:619-627.

Moreno-Casasola, P. 2006. Entornos veracruzanos: la costa de La Mancha. Instituto de Ecología, A.C., Xalapa, Ver. México, 576 pp.

Ortiz-Pulido, R., Laborde, J. \& Guevara., S. 2000. Frugivoría por aves en un paisaje fragmentado: Consecuencias en la Dispersión de Semillas. Biotropica 32:473-488.

Pech-Canche, J. M., Sosa-Escalante, J. E. \& Koyoc-Cruz, M. E. 2009. Guía para la identificación de pelos de guardia de mamíferos no voladores del estado de Yucatán, México. Revista Mexicana de Mastozoología 13: 7-33. 
Perea, R., Delibes, M. Polko M., Suárez-Esteban A., \& Fedriani J. M.. 2013. Context-dependent fruit-frugivore interactions: partner identities and spatio-temporal variations. Oikos 122:943-95.

Peredo, A., Martínez, D., Rodríguez-Pérez, J., and García D. 2013. Mammalian seed dispersal in Cantabrian woodland pastures: Network structure and response to forest loss. Basic and Applied Ecology 14:378-386.

Ramírez-Pinero, M. 2012. Técnicas para la restauración de la selva baja caducifolia en el centro de Veracruz. Tesis de Maestría. Instituto de Ecología, A.C. Xalapa, Veracruz.
Stoner, K. E., Riba-Hernández, P., Vulinec, K., \& Lambert, J. E. 2007. The role of mammals in creating and modifying seed shadows in tropical forests and some possible consequences of their elimination. Biotropica 39:316-327.

Zhou, Y., Zhang, L., Kaneko, Y., Newman, C. \& Wang, X. M. 2008. Frugivory and seed dispersal by a small carnivore, the Chinese ferret-badger, Melogale moschata, in a fragmented subtropical forest of central China. Forest Ecology and Management 255:1595-1603. 